\title{
Editorial: Special Issue on Computability, Complexity and Randomness
}

\author{
Noam Greenberg ${ }^{1}$
}

Published online: 24 July 2015

(C) Springer Science+Business Media New York 2015

This special issue of Theory of Computing Systems contains articles which relate to talks given at the 8th International Conference on Computability, Complexity and Randomness (CCR) in Moscow from the 23rd to the 27th of September 2015. The CCR conference series is devoted to algorithmic randomness, Kolmogorov complexity, and their relationship with computability theory, complexity theory, logic and reverse mathematics. The contributions to this special issue reflect both the interests of reserachers in Russia, in particular Kolmogorov and computational complexity, and new directions in the field of algorithmic randomness such as the focus on normal numbers and connections to ergodic theory. No conference proceedings have been published; this special issue is the only publication relating to the confernece. All contributions are a significant extension of the conference presentations, and underwent the usual rigorous journal reviewing process.

The article Polynomial-time algorithms for checking some properties of Boolean functions given by polynomials by Svetlana Selezneva and Anton Bukhman presents a polynomial time algorithm, which tests whether a Boolean function represented by a multivariate polynomial over $G F(2)$ is even, that is, has zero derivative in the direction of $(1, \ldots, 1)$. The approach is based on the notion of the characteristic of monomials in the polynomial representation of Boolean functions. In Normality and finite-state dimension of Liouville numbers Satyadev Nandakumar gives a new construction of a Liouville number which is normal for a given base.

Vladimir V'yugin in On Stability of Probability Laws with Respect to Small Violations of Algorithmic Randomness examines the robustness of ergodic theorems when the assumption of randomness is relaxed only a little. For example he shows that

Noam Greenberg

greenberg@msor.vuw.ac.nz

1 School of Mathematics, Statistics and Operations Research, Victoria University of Wellington, Wellington, New Zealand 
Birkhoff's ergodic theorem is not stable for all computable ergodic systems at once. The title The sum $2^{\mathrm{KA}(x)-\mathrm{KP}(x)}$ over all prefixes $x$ of some binary sequence can be infinite states the main result of Mikhail Andreev and Akim Kumok's; this solves a problem posed by J. Miller. Kenshi Miyabe continues his investigations into relativisations of Schnorr randomness in Reducibilities relating to Schnorr randomness. He introduces weak reducibilities modelled after those in the context of Martin-Löf randomness; this allows him to characterize computably traceable reducibility using relative Schnorr randomness, answering a questions of Nies'.

An algorithmic sufficient statistic of a string is a model that is supposed to contain all "useful" information in the string. However if it is well defined, it is weakly equivalent to an initial segment of Chaitin's Omega. In Algorithmic Minimal Sufficient Statistics: a New Approach, Nikolay Vereshchagin proposes a strong variant with better properties. Finally, in Relating and contrasting plain and prefix Kolmogorov complexity, Bruno Bauwens presents a short proof of Solovay's inequalities relating plain a prefix-free Kolmogorov complexities $C$ and $K$. His technique allows him to solve two open problems; for example, he answers a question of Bienvenu's by showing that there is a 2-random sequence with infinitely many initial segments with bounded plain deficiency but unbounded prefix-free deficiency.

I would like to thank the local organizing committee: Michael Raskin, Andrey Romashchenko, Alexander Shen and Nikolay Vereshchagin for staging a successful conference, the speakers, authors and referees for their work, the members of the program committee, and Alan Selman and the staff of Theory of Computing Systems for making this special issue possible. 